\title{
Determination of vancomycin minimum inhibitory concentration for ceftazidime resistant Streptococcus pneumoniae in Iran
}

\author{
Ramezan Ali Ataee ${ }^{1}$, Samira Habibian ${ }^{1}$, Ali Mehrabi-Tavana ${ }^{*}$, Zyanab Ahmadi², Nematollah Jonaidi
} and Mahmood Salesi ${ }^{4}$

\begin{abstract}
Background: In the context of growing health concerns over antibiotic resistance, the evaluation of the minimum inhibitory concentration (MIC) of vancomycin for Streptococcus pneumoniae (S. pneumoniae) strains resistant to ceftazidime becomes important for guiding health policy makers. The aim of this study was to determine vancomycin MIC of ceftazidime resistant S. pneumoniae strains.

Methods: Fifty identified serotypes of ceftazidime resistant S. pneumoniae strains were included in the study. The vancomycin MIC of the above mentioned bacteria was determined based on the 0.5 McFarland standards, by using a microdilution broth and the Etest method.

Results: The results showed that out of 50 ceftazidime resistant strains of S. pneumoniae, 46 strains (92\%) have shown a vancomycin MIC $\leq 0.19-0.1 .5 \mu \mathrm{g} / \mathrm{ml}$ and only four strains (8\%) have shown a vancomycin MIC equal to $1.5 \mathrm{\mu g} / \mathrm{ml}$ and the related maximum zone of inhibition was of 10 millimeter diameters.

Conclusions: The results of this investigation point out the emergence of $S$. pneumoniae strains with a vancomycin MIC $\geq 1.5 \mu \mathrm{g} / \mathrm{ml}$, which were resistant to ceftazidime. This finding uncovers a major health concern: a vancomycin MIC higher than $1.5 \mu \mathrm{g} / \mathrm{ml}$ and maximum zone of inhibition of only 10 millimeter. These findings represent an important warning for health authorities globally, concerning the treatment of patients, as the occurrence of S. pneumoniae strains with decreased vancomycin susceptibility has been demonstrated.
\end{abstract}

Keywords: Ceftazimdime resistance, Etest, Streptococcus pneumoniae, Vancomycin, Minimum inhibitory concentration, Maximum zone of inhibition

\section{Background}

Streptococcus pneumoniae (S. pneumoniae) is one of the major causes of invasive bacterial disease, such as pneumonia and meningitis, leading to increased morbidity and mortality rates, especially among the elderly and infants [1]. In addition, the results of a recent study have showed that $S$. pneumoniae is colonizing the nasopharynx and, on a single occasion, appropriate culturing yields pneumococci in $5-10 \%$ of healthy adults and $20-40 \%$ of healthy children. With regard to healthy carriers, children are at a

\footnotetext{
* Correspondence: Mehrab@bmsu.ac.ir

'Health Management Research Center, Baqiyatallah University of Medical Sciences, Tehran, Iran

Full list of author information is available at the end of the article
}

higher mortality risk compared to elderly and also, have an increased risk of pneumococcal infection. It has been shown that by repeated attempts and with the improvement of bacteriological methods, the percentage of nasopharyngeal carriers rises from $40 \%$ to $60 \%$, or greater, in all age groups [2]. This finding may be an explanation of the development of antibiotic-resistant strains. Because penicillin is the first drug of choice in the treatment of pneumococcal infections, yet the incidence of penicillin resistance in S. pneumoniae has increased dramatically, this phenomenon becomes a major health concern worldwide [3,4]. Results of a previous study have revealed that penicillin resistance rate was $65 \%$ in children, versus $22 \%$ in adults [5]. Furthermore, the increase of multidrug resistance in S. pneumoniae isolates makes the treatment of 
these infections even more difficult [6]. However, vancomycin is the last drug of choice in the case of pneumococcal meningitis caused by non- susceptible to penicillin strains and it has been used more frequently for severe, invasive infectious disease, where resistance to other antibiotics was present. This translates into the fact that vancomycin plays a key role in the management of multidrug-resistant pneumococcal infections [7]. These data emphasized antibiotic susceptibility surveillance systems are an important tool for preventing the emergence and spread of multidrug-resistant pathogens. Only limited data available suggest that imipenem and vancomycin may be the most active against penicillinresistant strains of $S$. pneumoniae $[8,9]$. However, the results of another study, in Serbia, indicated that multidrug resistance was found in one third of pneumococcal isolates, whereas all isolated pneumococcal strains were susceptible to vancomycin, linezolid, fluoroquinolones, telithromycin and rifampicin [1]. Nevertheless, vancomycin tolerant S. pneumoniae strains have been reported and characterized [10]. In order to determine vancomycin tolerance (VTSP) or vancomycin non susceptible of S. pneumoniae, continuous and comprehensive monitoring of minimum inhibitory concentration (MIC) may be successfully associated with the management and treatment [11]. Recently, based on research results, the ability to survive exposure to an antibiotic, through the loss of autolysin activity or triggering, is termed "tolerance" [12,13]. As a result, Normark $\mathrm{BH}$ and his colleagues showed the limiting criteria for pneumococcal vancomycin tolerance. They showed that the mean log kill value was three $(\mathrm{SD} \pm 0.3)$ and the mean loss of optical density (OD) was $89 \%$ (SD \pm 3 ). The results of these investigators' studies indicate that, for the pneumococcal lyt 4-4 strain, the log kill was 1.4 ( $\mathrm{SD} \pm 0.3$ ) and the loss of OD was 19\% (SD \pm 12 ). Based on these limitations, they have defined vancomycin tolerance as a $\log$ kill of $\leq 2$ and as OD loss of $\leq 43 \%$ [14]. As can be seen, the interpretation of data on vancomycin tolerance is very difficult and may be impossible for clinical laboratory technicians. However, recent data show that the MIC of the antibiotic has changed and it has been suggested that this tolerance could result in the development of drug resistance [15]. The initial therapy for S. pneumoniae infections based on accurate antibiotic resistance patterns among isolates in every region becomes a necessity and leads to a minimization of the development of antibiotic resistance [16]. The results of a previous study of our team revealed that $48 \%$ of $S$. pneumoniae strains were resistant to ceftazidime [17]. Therefore, specific and reliable studies are needed to detect and evaluate the vancomycin MIC among ceftazidime resistant pneumococcal clinical isolates in Iran. Therefore, the aim of this study was to determine vancomycin MICs, with the distribution of ceftazidime resistant S. pneumoniae serotypes in
Iranian patients, by using serial microdilution and the Etest method.

\section{Materials and Methods}

In this investigation, vancomycin MIC strips (MIC Test Strip, Liofilchem, Roseto degli Abruzzi Italy) were used. Serial microdilutions of native manufactured vancomycin $(500 \mathrm{mg} / \mathrm{vial}$, Dana Pharmacological Co, Tabriz, Iran) were performed, while Mueller-Hinton broth (Merck, Darmstadt, Germany), defibrinated lysed sheep blood (Baharafshan Co., Teheran, Iran), 96-well microplate (Technogen Spa, Pontenure Piacenza, Italy) and injectable distilled water were used.

\section{Bacterial strains}

A total of 50 clinical S. pneumoniae strains, with previously determined serotypes [1] and also resistant to ceftazidime, were subjected to vancomycin MIC determination. By using standard procedures recommended, lyophilized strains were recovered. In this investigation, antimicrobial susceptibility testing was performed according to Clinical and Laboratory Standards Institute (CLSI) guidelines [18]. The antimicrobial susceptibility patterns of the strains to vancomycin were determined by using both microdilution and the Etest method as confirmatory testing.

\section{Inoculums size}

Bacterial suspensions for inocula were prepared from 18 hours BHI broth medium equivalent to McFarland 0.5 standard, which provides turbidity comparable with that of a bacterial suspension containing $1.5 \times 10^{8}$ colonyforming units (CFU) $/ \mathrm{ml}$. In fact, a McFarland 0.5 standard corresponds to an OD 600 between 0.08 and 0.1 .

\section{Vancomycin minimum inhibitory concentration determination by etest method}

Each of the bacterial strains were separately inoculated on Muller-Hinton Agar plate (MHAP) containing 5\% defibrinated lysed sheep blood and a vancomycin MIC test strip (Liofilchem, Roseto degli Abruzzi, Italy) was placed on the inoculums lawn. Briefly, the procedure was performed as follows: a sterile swab was sunk into the bacterial suspension and then inocula onto MullerHinton 5\% defibrinated sheep blood agar plates and after 15 to $20 \mathrm{~min}$, when the bacterial suspension were absorbed onto the medium, vancomycin MIC strips were applied on the plate. The plates were incubated at $37^{\circ} \mathrm{C}$ for $24 \mathrm{~h}$ in an atmosphere containing 3-5\% $\mathrm{CO}_{2}$.

After the incubation period, the elliptical zone of inhibition was measured and the MIC of each strain was determined, separately. The MIC was the point where the elliptical zone of growth inhibition intersected the MIC scale on the vancomycin strip. The antibiotic gradient scale on the vancomycin MIC strip was $0.016-256 \mu \mathrm{g} / \mathrm{ml}$. 


\section{Vancomycin minimum inhibitory concentration determination by serial microdilutions method}

Based on our previous reports and references of bacterial strains applied [19], the stock solution of vancomycin was prepared. Serial concentrations with twice the anterior value progressive rate $(0.5,1,2,4,8,16,32,64,128 \mu \mathrm{g} /$ well $)$ of vancomycin were then prepared and loaded into defined rows of 96 well microplates from the antibiotic stock solution. Plates were incubated at $35^{\circ} \mathrm{C}$ for 24 hours, until evaporation and finalization of the process of loading the antibiotic into the plate. The plates were stored in a refrigerator at $4^{\circ} \mathrm{C}$ for 3 hours.

The designing of the 96-well microplate (for preparation of the serial dilutions of vancomycin) was performed as follows: one row for negative control, one row for positive control and three rows for tests were marked, respectively. Therefore, each 96-well microplate was considered to measure the antibiotic sensitivity of two bacterial strains. The negative control rows for each case consisted of the serial dilutions of vancomycin $(0.5,1$, $2,4,8,16,32,64,128 \mu \mathrm{g} /$ well), culture media (MuellerHinton broth containing 3\% defibrinated sheep blood) and no bacterial suspension. The positive control rows consisted of culture media (Mueller-Hinton broth containing $3 \%$ defibrinated sheep blood) and the bacterial suspension. The positive control rows were lacking vancomycin. The three rows of microplates for tests with equal conditions were used for each bacterial strain testing. The test rows were composed of serial dilutions of vancomycin $(0.5,1,2$, $4,8,16,32,64,128 \mu \mathrm{g} /$ wells, respectively), culture media and bacterial suspension. After inoculation and incubation, the plates were subjected to scanning at $450 \mathrm{~nm}$ of wavelength ELISA reader. Data were analyzed using unilateral analysis of variance (ANOVA). In our statistical analyses, $\alpha=0.05$ was considered acceptable for significant variation and the results were analyzed using SPSS Version 16.0 Software (SPSS Inc., Chicago, IL, The USA).

\section{Molecular assay for resistance gene}

In this study, we used previously published collections of primer pairs [20] or designed them based on the nucleotide reference GeneBank. Polymerase chain reactions (PCR) for $P B P 2 b, P B P 2 x, \operatorname{gyr} A$, vex2 and $v n c S$ genes, which amplified 1500, 1920, 2100, 400 and 1000 bp fragments were performed respectively (Table 1).

In our study, the mentioned primer pairs were selected and synthesized by CinnaGen Co, Teheran, Iran. For the detection and amplification of the above mentioned genes, overnight grown bacterial cells of the late exponential phase were subjected to DNA extraction, using the salting out method [21]. The $25 \mu \mathrm{L}$ volume PCR reaction master mixes were prepared in $0.2 \mathrm{~mL}$ microcentrifuge tubes. An individual reaction was carried out as follows: $2.5 \mu \mathrm{L}$ PCR buffer (10x), $\mathrm{MgCl}_{2}$ (50-150 mM gradient), $0.5 \mu \mathrm{L}$ dNTPs (10 mM), $0.5 \mu \mathrm{L}$ of each primer (100 pm/ $\mu \mathrm{l}), 0.5 \mu \mathrm{L}$ Taq DNA polymerase (5 unit/ $\mu \mathrm{l})$, $1.5 \mu \mathrm{L}$ template DNA and $18.5 \mu \mathrm{L}$ sterile deionized water. All the materials used in PCR reactions were purchased from CinnaGen Co., Teheran, Iran. Thermal cycling was performed in the Analytic Jena PCR system (Analytic Jena AG, Jena, Germany). The PCR process included denaturalization at $94^{\circ} \mathrm{C}$ for $6 \mathrm{~min}$, followed by 35 amplification cycles of $94^{\circ} \mathrm{C}$ for $45 \mathrm{~s}, 61^{\circ} \mathrm{C}$ for $30 \mathrm{~s}, 72^{\circ} \mathrm{C}$ for $1 \mathrm{~min}$ and with $5 \mathrm{~min}$ final extension at $72^{\circ} \mathrm{C}$. For the analysis of the PCR products, $1.5 \%$ agarose gel containing $1 \mathrm{X}$ TBE buffer was prepared and was run at $100 \mathrm{~V}$ for $40 \mathrm{~min}$. For determining the size of the PCR products, we have used a $50 \mathrm{bp}$ DNA ladder.

\section{Results}

Demographic analysis revealed that the mean age of patients participating in this study was 52 years old (16 to 73 years old), of which $30(60 \%)$ were women and 20 (40\%) were men.

\section{The results of vancomycin susceptibility pattern}

The results of broth microdilution method with vancomycin gradient (concentration) ranges of $0.5,1,2,4,8$, $16,32,64,128 \mu \mathrm{g} /$ well revealed that 46 isolates $(92 \%)$ with $\mathrm{MIC} \leq 1.5 \mu \mathrm{g} /$ well were obtained. Four strains $(8 \%)$ with $\mathrm{MIC} \geq 1.5 \mu \mathrm{g} /$ well, one of them with a MIC $=8 \mu \mathrm{g} / \mathrm{well}$ (Figure 1), were detected. The results of vancomycin MIC determined by the Etest and broth microdilution method for these strains were the same. In fact, the comparative results of broth microdilution and Etest method revealed 46 isolates (92\%) with a $\mathrm{MIC} \leq 1.5 \mu \mathrm{g} / \mathrm{ml}$, three strains (6\%) with $\mathrm{MIC} \geq 1.5 \leq 6 \mu \mathrm{g} / \mathrm{ml}$ and one strain (2\%) with MIC $8 \mu \mathrm{g} / \mathrm{ml}$. However, analysis of the Etest results indicated that the lowest MIC, was $0.125 \mu \mathrm{g} / \mathrm{ml}$, belonged to serotype 4, followed by serotypes 6 and 5, which were isolated from different wounds, while three strains from the lungs, with a $\mathrm{MIC}=0.25-0.75 \mu \mathrm{g} / \mathrm{ml}$ and one strain with a $\mathrm{MIC}=0.38 \mu \mathrm{g} / \mathrm{ml}$, isolated from the blood, belonged to serotype 1 (Table 2).

Figure 1 shows the example of a 24 hours incubation period of a 96-well microplate used for measuring the antibiotic sensitivity of two bacterial strains. The results of the subculturing of each well showed that the vancomycin MIC for S. pneumoniae strains was the same as of the Etest strip results, which are displayed in Table 2. The unilateral ANOVA of the OD of each well at time 0 and 24 hours after incubation of the bacterium with different concentrations of antibiotics per well have shown the significant differences $(\mathrm{P} \leq 0.02)$.

The results of this study revealed that the vancomycin MIC of $92 \%$ of pathogenic S. pneumoniae is $0.19-$ $1.5 \mu \mathrm{g} / \mathrm{ml}$, as a vancomycin susceptible strain, while $8 \%$ of them have a vancomycin MIC $\geq 1.5 \mu \mathrm{g} / \mathrm{ml}$, being 
Table 1 The sequences of the primer pairs used in this study

\begin{tabular}{|c|c|c|c|c|}
\hline \multirow[t]{2}{*}{ Gene } & \multirow[t]{2}{*}{ Nucleotide sequences } & \multicolumn{3}{|l|}{ Charactristics } \\
\hline & & Size of amplified product (bp) & Induce resistant to & Reference \\
\hline \multirow[t]{2}{*}{ PBP2b } & F - 5' GAT CCT CTA AAT GAT TCT CAG GTG G 3' & $1500 \mathrm{bp}$ & Penicillin & {$[20]$} \\
\hline & R - 5' TGG TGT TCG TGT GGC TCC TC 3 & & & \\
\hline \multirow[t]{2}{*}{$P B P 2 x$} & F- 5' GAT TGC TGA GGA TGC AAC CTC TTA TAA TGT CTA TG 3' & $1920 \mathrm{bp}$ & Ceftazidime & \\
\hline & R -5' GCC TTG AAA TTC AAG TTC TAT ATT GAG CCA CTT AGC 3' & & Ceftriaxon & \\
\hline \multirow[t]{2}{*}{ gyrA } & F - 5' CCG TTG TGA AAG TCA CTA TCT G 3' & $2100 \mathrm{bp}$ & Ciprofloxacin & In this study \\
\hline & R - 5' AGT TGC TCC ATT AAC CA 3' & & & \\
\hline \multirow[t]{2}{*}{ vncs } & F - 5' AAA CAA TTG ACC TCC TCC AGA TG 3' & $1000 \mathrm{bp}$ & Vancomycin & \\
\hline & R $-5^{\prime}$ TTC TAA CTC CAT CTA TGT AAA CC $3^{\prime}$ & & & \\
\hline
\end{tabular}

considered with decreased susceptibility to vancomycin or tolerant, which belonged to serotype 17 and was isolated from the blood.

The results of the susceptibility pattern obtained in this study have shown that the most frequent $S$. pneumoniae strain with sensitivity to vancomycin was serotype 6 , isolated from lung. In Figure 2, rows $\mathrm{A}-\mathrm{C}$ showed the three Ceftazidime resistant $S$. pneumoniae strains with MICs of 6,12 and $32 \mu \mathrm{g} / \mathrm{ml}$, respectively, while part D and E showed the two S. pneumoniae strains with decreased vancomycin sensitivity, with a MIC $\geq 1.5 \mu \mathrm{g} / \mathrm{ml}$.

The key finding of this study is shown in the last column of Table 2. By comparing the obtained growth zone of inhibition, we can estimate the vancomycin susceptibility rate or tolerance of the bacterial strains. For example, the vancomycin MIC for S. pneumoniae serotype 4 was $0.125 \mu \mathrm{g} / \mathrm{ml}$, while vancomycin MIC for serotypes
17 and 20 were 8 and $6 \mu \mathrm{g} / \mathrm{ml}$, respectively, with the same zone of inhibition.

\section{Results of molecular assay}

The results of the molecular assay for $v n c S$ and vex2 genes revealed that the used primer pairs in this study were not able to amplify the coding sensor histidine kinase sequences as vancomycin tolerant in all 50 isolated strains of $S$. pneumoniae, while the results of PCR for $P B P 2 b, P B P 2 x$ and gyrA, as determinants of penicillin, ceftriaxone, ceftazidime and ciprofloxacin resistance, amplified the fragment of their related genes (Figure 3). On the other hand, the PCR protocol shows no vancomycin tolerant genes in the studied S. pneumoniae strains.

The outlined results of this study revealed that the vancomycin MIC of $92 \%$ (46 strains) of pathogenic $S$. pneumoniae is $0.19-1.5 \mu \mathrm{g} / \mathrm{ml}$, while $8 \%$ (four strains)

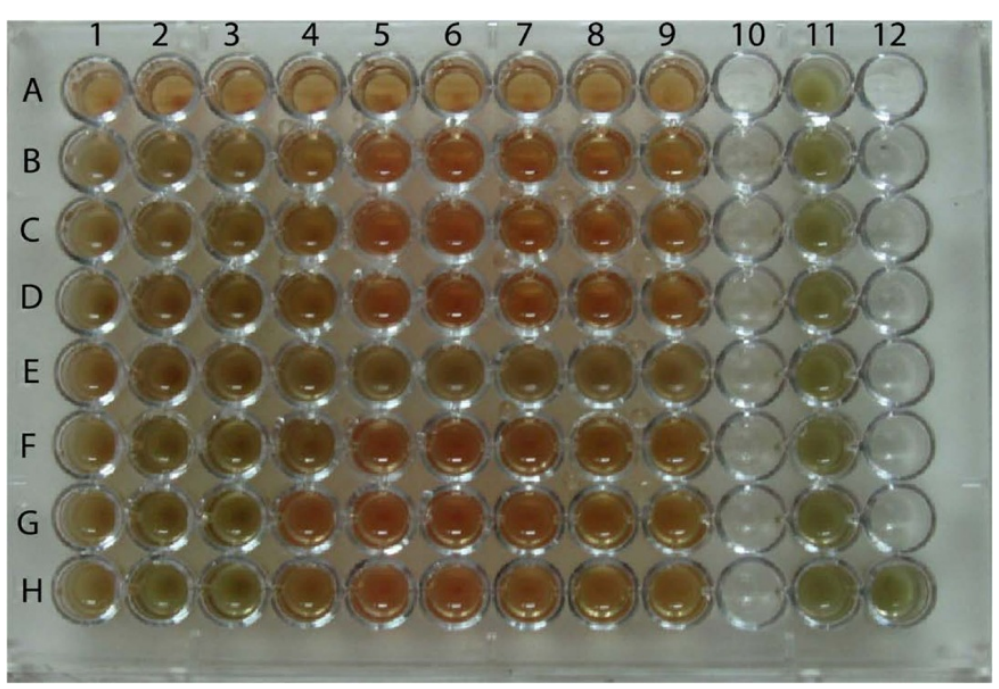

Figure 1 Illustration an example of the 96 well micro plate's broth microdilution method for two S. pneumoniae strains vancomycin assay. In this plate the 9 wells in $\mathbf{A}$ row is negative control and two three test rows (rows $\mathbf{B}, \mathbf{C}$ and $\mathbf{D}$ for strain one and the rows $\mathbf{F}, \mathbf{G}$ and $\mathbf{H}$ for strain two) for vancomycin MIC determination which contain the serial concentration of 0.5 to $128 \mu \mathrm{g} /$ well of vancomycin respectively. Nine wells of $\mathbf{E}$ row and well on column 11 are marked as positive control. 
Table 2 Results of S. pneumoniae serotype distribution and vancomycin MIC by Etest strip and broth microdilution method, containing vancomycin gradients of $0.016-256 \mu \mathrm{g} / \mathrm{ml}$ and $0.5-128 \mu \mathrm{g} / \mathrm{well}$ respectively

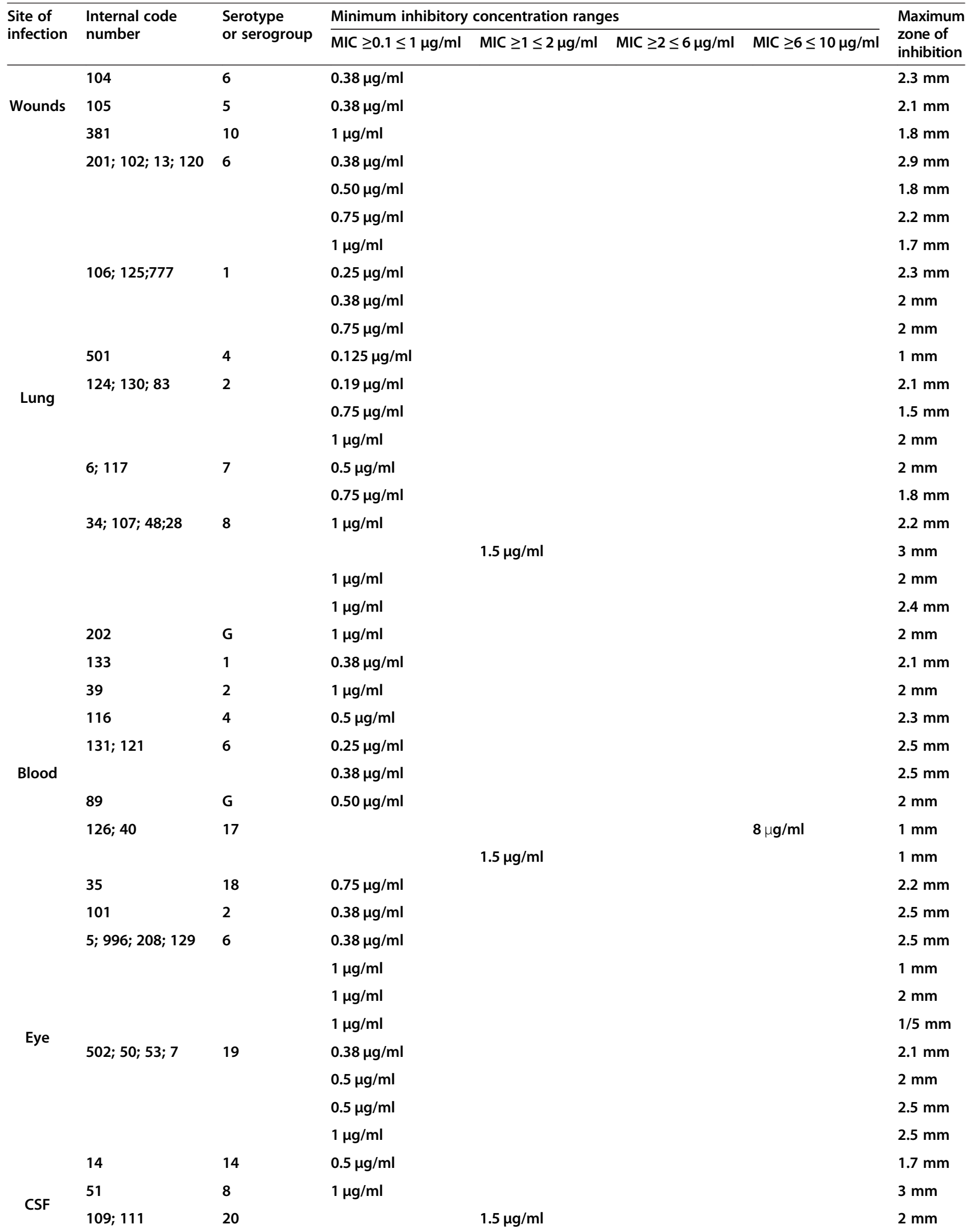


Table 2 Results of S. pneumoniae serotype distribution and vancomycin MIC by Etest strip and broth microdilution method, containing vancomycin gradients of $0.016-256 \mu \mathrm{g} / \mathrm{ml}$ and $0.5-128 \mu \mathrm{g} / \mathrm{well}$ respectively (Continued)

\begin{tabular}{|c|c|c|c|c|c|}
\hline & & & & $6 \mu \mathrm{g} / \mathrm{ml}$ & $1 \mathrm{~mm}$ \\
\hline \multirow{4}{*}{ Throat } & 128 & 7 & $1 \mu \mathrm{g} / \mathrm{ml}$ & & $2 \mathrm{~mm}$ \\
\hline & 123 & 20 & & $4 \mu \mathrm{g} / \mathrm{ml}$ & $1 \mathrm{~mm}$ \\
\hline & 103 & 4 & $0.38 \mu \mathrm{g} / \mathrm{ml}$ & & $2.4 \mathrm{~mm}$ \\
\hline & 108 & 14 & & $5 \mu \mathrm{g} / \mathrm{ml}$ & $1 \mathrm{~mm}$ \\
\hline \multirow{2}{*}{ Nostrils } & $778 ; 49$ & 7 & $0.5 \mu \mathrm{g} / \mathrm{ml}$ & & $1.5 \mathrm{~mm}$ \\
\hline & & & $0.5 \mu \mathrm{g} / \mathrm{ml}$ & & $2.5 \mathrm{~mm}$ \\
\hline Sinus & 115 & 20 & $1 \mu \mathrm{g} / \mathrm{ml}$ & & $1.7 \mathrm{~mm}$ \\
\hline
\end{tabular}

have a vancomycin $\mathrm{MIC} \geq 1.5 \mu \mathrm{g} / \mathrm{ml}$. The related genes resistant to penicillin, ceftriaxone, ceftazidime were detected in several $S$. pneumoniae strains, which had been marked as $\beta$-lactam resistant isolates.

\section{Discussion}

Pneumococcal resistance to antibiotics, especially to penicillin, poses an increased therapeutic concern [22,23]. In addition, vancomycin tolerant $S$. pneumoniae had been observed in three clinical isolates in the early '90s [7]. However, given the fact that the antibiotic of choice for treating meningeal infections caused by penicillin resistant $S$. pneumoniae strains is vancomycin and also, empirical therapy for purulent meningitis caused by head trauma or post neurosurgery are an indication for vancomycin plus either ceftazidime, cefepime or meropenem, researchers have recommended the necessity to assay the efficiency of vancomycin in treating infections caused by $S$. pneumoniae $[14,24]$. Furthermore, although tolerances to vancomycin were obtained based on accurate experimental studies, the determination and interpretation of vancomycin tolerance is laborious and may pose difficulties for medical diagnostic
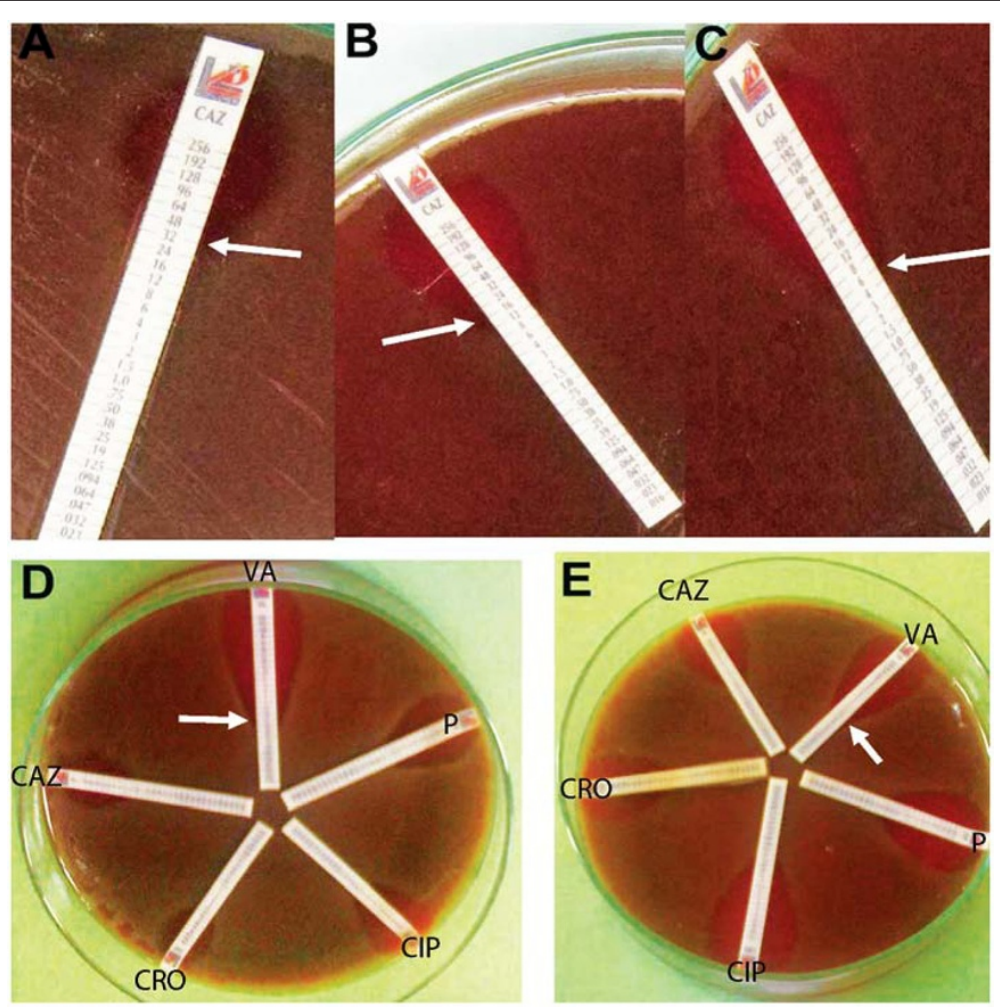

Figure 2 Sections A - C show the elliptical zone of inhibition of three S. pneumoniae strains around ceftazidime MIC strip, with MIC =32, 16 and $6 \mu \mathrm{g} / \mathrm{ml}$ of ceftazidime, respectively. In Part $\mathbf{D}$ and $\mathbf{E}$, antibiotic susceptibility assay plates using Etest strips for penicillin, vancomycin, ceftazidime, ceftriaxone and ciprofloxacin are shown for two S. pneumoniae strains. Arrays in part $\mathbf{D}$ and $\mathbf{E}$ are shown the vancomycin $\mathrm{MIC}=1.5$ and 4 $\mu \mathrm{g} / \mathrm{ml}$ respectively. 


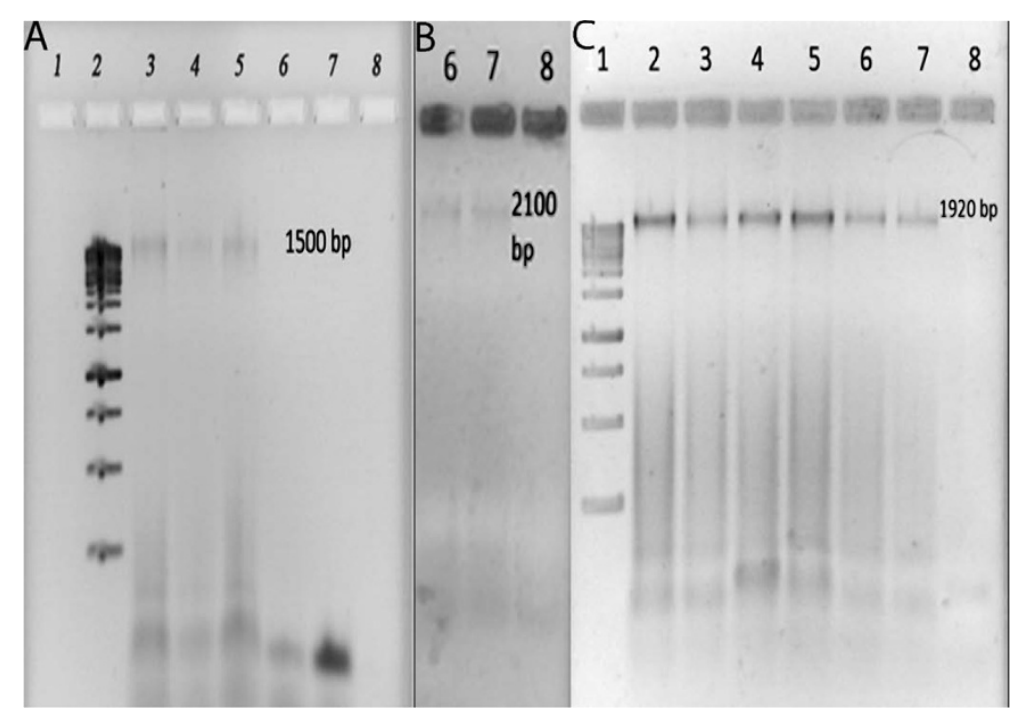

Figure 3 Sections A, B and C depicts the amplified 1500, 1920 and 2010bp fragments of PBP2b, PBP2x and gyrA that introduces resistance to penicillin, ceftazidime and or ceftriaxone and ciprofloxacin.

laboratories. The results of the present study have revealed that the Etest and microdilution methods for determination vancomycin MIC can be easily performed and are predictive indicators for the tolerance or decrease of susceptibility to vancomycin.

In this regards, vancomycin MIC determination for pathogenic ceftazidime resistant S. pneumoniae strains has not been reported up to present. The results of vancomycin MIC determination of different S. pneumoniae serotypes were shown in Table 2 . The MIC of vancomycin for strains belonging to serotypes 2 and 4 revealed a maximum zone of inhibition of 21 and 10 millimeter in diameter, respectively. These two serotypes were isolated from the lung. The vancomycin MIC of 10 strains (20\% of isolates) (serotypes 1, 2, 4, 5, 6, and 19) was equal to $0.38 \mu \mathrm{g} / \mathrm{ml}$ of vancomycin, with a zone of inhibition of 20-29 millimeters in diameter. The MIC of 14 different serotypes (28\% of isolates) were equal to $0.5-0.75 \mu \mathrm{g} / \mathrm{ml}$ of vancomycin, with a zone of inhibition of 15-25 millimeter in diameter. The MIC for other 14 different serotypes (28\% of isolates) was equal to $1 \mu \mathrm{g} / \mathrm{ml}$ of vancomycin, corresponding to a zone of inhibition of 10-30 millimeters in diameter. Three serotypes (6\% isolates) have MICs equal to $1.5 \mu \mathrm{g} / \mathrm{ml}$, with a zone of inhibition of $1-3$ centimeters in diameter. Two isolates (4\%) of the serotype 20 had a MIC of 4 and $6 \mu \mathrm{g} / \mathrm{ml}$, while the MICs of serotypes 14 and 17 (4\%) were 5 and $8 \mu \mathrm{g} / \mathrm{ml}$, respectively, with a zone of inhibition equal to 10 millimeter in diameter. According to the CLSI guidelines, a MIC of $2 \mu \mathrm{g} / \mathrm{ml}$ of vancomycin, with a zone of inhibition $\leq 17$ millimeters should be regarded as a resistant strain [18]. However, the sensitive ranges of a vancomycin MIC is less $2 \mu \mathrm{g} / \mathrm{ml}$. It can be suggested that in this study 46 S. pneumoniae strains (92\%) were vancomycin susceptible, while four strains (8\%) manifested a decreasing susceptibility to vancomycin.

A very important finding of the current study was that the isolates presenting a decreasing susceptibility to vancomycin were also resistant to ceftazidime. In addition, the results of our previous study showed that nine S. pneumoniae strains (18\%) were resistant to penicillin [19]. The results of this study indicate that only four out of 50 ceftazidime resistant of S. pneumoniae strains manifested decreased susceptibility to vancomycin.

In 2010, Moscoso et al. showed that the vancomycin tolerance in S. pneumoniae isolates depends on reduced enzyme activity of the major LytA autolysin or capsular polysaccharide [11]. In this study, the PCR methods were not able to determine the $\operatorname{van} \mathrm{A}$ or $\operatorname{van} \mathrm{B}$ gene, which induced vancomycin resistance. Despite the present study, Kohanteb and Sadeghi (2007) reported that vancomycin MIC for penicillin-resistant S. pneumoniae strains in Iran was $0.03-0.5 \mu \mathrm{g} / \mathrm{ml}$ [25]. Bokaeian et al. (2011) reported that the vancomycin MIC of S. pneumoniae from nasopharyngeal carriage among healthy adolescents in Zahedan was $0.02-1 \mu \mathrm{g} / \mathrm{ml}$, and concluded that all S. pneumoniae isolates were susceptible to vancomycin [26]. However, Sanaei DA et al., which studied the nasopharyngeal carrier rate of S. pneumoniae in children by using the disc diffusion method, showed that $1.5 \%$ of S. pneumoniae isolates were non susceptible to vacomycin [27].

\section{Conclusion}

However, have not access to positive and negative control strains was the main limitation of this investigation. the results of this study revealed that $92 \%$ of pathogenic 
ceftazidime resistant strains of $S$. pneumoniae have vancomycin MICs of $0.19-1.5 \mu \mathrm{g} / \mathrm{ml}$ and only $8 \%$ of them have a vancomycin MIC of $4-8 \mu \mathrm{g} / \mathrm{ml}$. Based on the data collected from this research, the later serotypes had been marked as vancomycin tolerant or may be vancomycin non- susceptible strains. However, more ample genetic and molecular research are needed, as these findings represent an important warning to health authorities because the occurrence of S. pneumoniae strains with multiple resistances has been shown, a situation which has never been reported before.

\section{Competing interests}

The authors declare that they have no competing interests.

\section{Authors' contributions}

AMT and RAA have developed the original design of the study. RAA, SH and ZA have performed the experimental protocol and composed the abstract. Mahmode Salesi performed the analysis of data. RAA, SH and AMT wrote and prepared the final manuscript. All authors read and approved the final manuscript.

\section{Acknowledgements}

The authors would like to thank the head of Baqiyatallah Research Institute, Teheran, Iran, Dr. Mostafa Ghanei, for encouraging the authors in their efforts and English editor Kowsar edit Institute service CO, Tehran Iran.

\section{Author details}

'Health Management Research Center, Baqiyatallah University of Medical Sciences, Tehran, Iran. ${ }^{2}$ Molecular Biology Research Center, Baqiyatallah University of Medical Sciences, Tehran, Iran. ${ }^{3}$ Department of Infection Diseases and Health Research Center, Baqiyatallah University of Medical Sciences, Tehran, Iran. ${ }^{4}$ Department of Statistic and Health Research Center, Baqiyatallah University of Medical Sciences, Tehran, Iran.

Received: 7 July 2014 Accepted: 24 October 2014

Published online: 11 November 2014

\section{References}

1. Mehrabi Tavana A, Ataee RA, Esmaili D: Serotyping distribution of invasive pneumococcal disease (IPD) in Iranian patients. J Pure And Applied Microbiology 2012, 6(1):155-160.

2. Dudley S, Ashe K, Winther B: Bacterial pathogens of otitis media and sinusitis: detection in the nasopharynx with selective agar media. I Lab Clin Med 2001, 138:338-342.

3. Cardoso MR, Nascimento-Carvalho CM, Ferrero F: Penicillin-resistant pneumococcus and risk of treatment failure in pneumonia. Arch Dis Child 2008, 93(3):221-225.

4. Watanabe $Y$, Akizuki T: Prevention and treatment of penicillin-resistant Streptococcus pneumoniae meningitis after intracraniofacial surgery with distraction osteogenesis. J Craniofac Surg 2008, 19(6):1542-1548.

5. Jiang HC, Kui LY, Huang HL, Su M, Wen BP: Invasive isolates of Streptococcus pneumoniae in Serbia: antimicrobial susceptibility and serotypes. Sro Arh Celok Lek 2013, 141(1-2):48-53.

6. Shakya G, Adhikari BR: Ten-years surveillance of antimicrobial resistance pattern of Streptococcus pneumoniae in Nepal. Afr J Microbiol Res 2012, 6(20):4233-4238.

7. Novak R, Henriques B, Charpentier E, Normark S, Tuomanen E: Emergence of Vancomycin tolerance in Streptococcus pneumoniae. Nature 1999, 399:590-593.

8. Ateda K, Takashima K, Miyazaki H, Matsumoto T, Hatori T, Yamaguchi K: Noncompromised penicillin-resistant Pneumococcal pneumonia CBA/J mouse model and comparative efficacies of antibiotics in this model. Antimicrob Agents Chemother 1996, 40(6):1520-1525.

9. Jiang $H C$, Kui $L Y$, Huang $H L$, Su M, Wen BP: Frequency distribution and antibiotic resistance of pathogens from the cerebrospinal fluid of 116 children with bacterial meningitis. Zhongguo Dang Dai Er Ke Za Zhi 2013, 15(4):264-267.
10. Gillis LM, White HD, Whitehurst A, Sullivan DC: Vancomycin-tolerance among clinical isolates of Streptococcus pneumoniae in Mississippi during 1999-2001. Am J Med Sci 2005, 330(2):65-68.

11. Moscoso M, Domenech M, Garcia E: Vancomycin tolerance in clinical and laboratory Streptococcus pneumoniae isolates depends on reduced enzyme activity of the major LytA autolysin or cooperation between CiaH histidine kinase and capsular polysaccharide. Mol Microbiol 2010, 77(4):1052. $-1-64$

12. Sung $H$, Shin HB: Vancomycin-tolerant Streptococcus pneumoniae in Korea. J Clin Microbiol 2006, 44:3524-3528.

13. Hidalgo M, Castaneda $E$, Arias CA: Tolerance to vancomycin in a multiresistant, Colombian isolate of Streptococcus pneumoniae. J Antimicrob Chemother 2003, 52:300-302.

14. Normark Henriques B, Novak R, Ortqvist A, Kallenius G, Tuomanen E, Normark S: Clinical isolates of Streptococcus pneumoniae that exhibit tolerance of vancomycin. Clin Infect 2001, 32:552-558.

15. Kun k: Vancomycin Tolerance in Streptococcus pneumoniae. M.Sc thesis. Medical University of Hong Kong, Department of Microbiology; 2002.

16. Oliver A, Tarrago D, Martinez-Ferrer M, Loza E, Canton R, Baquero F: Comparison of the microdilution method (PASCO), the Etest and disc-diffusion in Streptococcus pneumoniae antibiotic susceptibility testing. Rev Esp Quimioter 1998, 11(4):344-348.

17. Habibian S, Mehrabi-Tavana A, Ahmadi Z, Izadi M, Jonaidi N, Darakhshanpoure J, Salesi M, Zahraei SM, Ataee RA: Serotype distribution and antibiotics susceptibility pattern of Streptococcus pneumonia in Iran. IRCMJ 2013, 15(10):e8053.

18. CLSI M100-S20: Performance Standard for Antimicrobial Susceptibility Testing. 2010, 30. No.1 is available in: http://f3.tiera.ru/1/genesis/575-579/ 575000/11b141afbf83788d000b56139415f744.

19. Ataee RA, Mehrabi-Tavana A, Hosseini SMJ, Morid K, Ghorbananli Zadegan M: A method for antibiotic susceptibility testing: applicable and accurate. Jundishapur J Microbiol 2012, 5(1):341-345.

20. Maren KR, Sogstad E, Høiby A, Caugant DA: Molecular Characterization of Non-Penicillin-Susceptible Streptococcus pneumoniae in Norway. J Clin Microbiol 2006, 44(9):3225-3230.

21. Sambrook J, Russel DW: Molecular Cloning: A Laboratory Manual. 3rd edition. New York: Coldspring Harbour Laboratory Press; 2001:417-421.

22. Baquero F: Pneumococcal resistance to b-lactam antibiotics: a global geographic overview. Microb Drug Resist 1995, 1:115-120.

23. Friedland $I R$, Shelton S, Paris M: Dilemmas in diagnosis and management of cephalosporin-resistant Streptococcus pneumoniae meningitis. Pediatr Infect Dis J 1993, 12:196-200.

24. Viladrich PF, Gudiol F, Linares J, Pallares R, Sabate I, Rufi G, Ariza J: Evaluation of Vancomycin for therapy of adult Pneumococcal meningitis. Antimicrob Agents Chemother 1991, 35:2467-2472.

25. Kohanteb J, Sadeghi E: Penicillin-resistant Streptococcus pneumoniae in Iran. Med Princ Pract 2007, 16(1):29-33.

26. Bokaeian M, Khazaei HA, Javadimehr M: Nasopharyngeal carriage, antibiotic resistance and serotype distribution of streptococcus pneumoniae among healthy adolescents in Zahedan. Iran Red Crescent Med J 2011, 13(5):328-333.

27. Sanaei DA, Abdinia B, Karimi A: Nasopharyngeal carrier rate of Streptococcus pneumoniae in children: serotype distribution and antimicrobial resistance. Arch Iran Med 2012, 15(8):500-503.

\section{doi:10.1186/s12941-014-0053-1}

Cite this article as: Ataee et al:: Determination of vancomycin minimum inhibitory concentration for ceftazidime resistant Streptococcus pneumoniae in Iran. Annals of Clinical Microbiology and Antimicrobials 2014 13:53. 